\title{
LINGUAGEM DE PROGRAMAÇÃO VISUAL DENTRO DO CONTEXTO BIM: MAPEAMENTO SISTEMÁTICO DA LITERATURA
}

\author{
Natalia Oliveira $^{(1)}$, Michele Carvalho ${ }^{(1)}$ \\ (1) Universidade de Brasília, Brasília
}

\begin{abstract}
Resumo
O conceito do Building Information Modeling (BIM) vem ganhando interesse cada vez maior na indústria da construção, pois viabiliza a criação de um modelo de informações da edificação durante todo o seu ciclo de vida, além de auxiliar no processo de tomada de decisões. A maioria dos softwares BIM oferece ao usuário a capacidade de adicionar funções personalizadas de modo a expandir os recursos do modelo. Uma das formas de se fazer isto é por meio da linguagem de programação visual (VPL). O BIM associado ao VPL permite aos usuários acessar de modo mais fácil e eficiente os dados da construção, a partir da criação de algoritmos de trabalho que sejam personalizados, flexíveis e poderosos. Deste modo, o objetivo do artigo é investigar, por meio do Mapeamento Sistemático da Literatura (MSL), o cenário atual da construção civil no que diz respeito à utilização da VPL vinculada ao BIM. A partir deste estudo, foi possível identificar que a implementação do VPL juntamente com a modelagem da informação possui grandes potencialidades e uma gama grande de aplicações, mas que ainda foram pouco exploradas, sendo o principal foco dos estudos a análise de alternativas de design da edificação nas etapas iniciais de projeto.
\end{abstract}

\section{Introdução}

Este artigo tem como objetivo investigar o panorama atual no que se refere a utilização da linguagem de programação visual (VPL) dentro do contexto do Building Information Modeling (BIM), em português, Modelagem da Informação da Construção, tentando identificar e caracterizar as principais aplicações do BIM-VPL dentro da indústria da arquitetura, engenharia e construção (AEC) e se existem benefícios ao se aplicar de forma conjunta estas duas ferramentas.

De modo geral, estudos mostraram como é vantajosa a utilização das tecnologias computacionais para auxiliar nas diferentes etapas do processo construtivo. Estas ferramentas são conhecidas por acelerar o processo de projeto e otimizar o desempenho de construção com custo relativamente baixo, auxiliando na tomada de decisões e na melhora de qualidade das atividades desenvolvidas. 
O BIM é definido pela ISO 29481 [1] como sendo uma representação digital compartilhada das características físicas e funcionais de qualquer objeto construído que forma uma base confiável para decisões. Ele permite que seja criado um modelo de informações da edificação durante todo o seu ciclo de vida, já que além da geometria da construção, contém numerosas informações sobre seus diferentes aspectos. Com uma gama de informações muito mais ampla, as funcionalidades dos modelos desenvolvidos no ambiente BIM também são ampliadas, permitindo sua utilização em todas as fases do ciclo de vida da edificação, indo desde os estudos de viabilidade, passando pelo desenvolvimento do projeto, simulações, planejamento, orçamentação, construção e controle, até se chegar á fase de uso, manutenção, reformas e, por fim, demolição e/ou reconstrução e/ou desconstrução [2].

Outro ponto importante do BIM diz respeito as suas dimensões (forma como os modelos estão programados), onde "Quanto mais dimensões tiver o modelo, maiores serão os tipos de informações possíveis de serem modeladas a partir deles, tornando as tomadas de decisão mais complexas e acertadas." [3]. Vários são os benefícios obtidos ao se adotar o BIM, uma vez que ele pode dar suporte e incrementar muitas práticas do setor construtivo. Além disso, a maioria dos pacotes de software BIM oferece ao usuário a capacidade de adicionar parâmetros ou funções personalizadas para aumentar o número de informações e expandir os recursos do modelo baseado em BIM [4].

Uma das formas de se expandir estes recursos é por meio do VPL. De acordo com Leitão e Santos [5] a linguagem de programação pode ser entendida como um meio formal para expressar ideias e não apenas uma maneira de fazer com que um computador realize operações. Esta linguagem pode ser do tipo gráfica/visual onde através de uma interface visual o programador conecta nós predefinidos que possuem entradas e saídas e que uma vez conectados uns aos outros, em uma sequência lógica, geram como resultado um script, ou seja, uma ação no software. O VPL permite que o usuário trabalhe dentro de um processo visual gerando algoritmos customizados que podem ser utilizados em uma grande quantidade de aplicações, que vão desde o processamento de dados até a geração de geometria [6].

De acordo com Leitão e Santos [5] algumas das vantagens da programação visual em relação as programações textuais mais antigas são o fato do VPL necessitar de uma quantidade de conhecimento menor e possuírem um ambiente de desenvolvimento mais amigável. Em decorrência destas facilidades, na última década aumentou o número de profissionais da construção com interesse pela ferramenta, uma vez que estas permitem criar algoritmos de trabalho que sejam personalizados, flexíveis e poderosos sem a necessidade de precisar aprender a escrever códigos de programação para isso [7].

\section{Metodologia}

O método de análise escolhido para conduzir este estudo foi o Mapeamento Sistemático da Literatura (MSL). Este método permite identificar, de modo sistematizado, os trabalhos e pesquisas que vem sendo desenvolvidos pela comunidade. Agostinho e Granja [8] definem cinco etapas para o desenvolvimento do MSL: definição das palavras-chave de busca, escolha das bases de dados, formulação da string de busca (sequência de caracteres de busca), critérios de seleção dos artigos e a extração das informações. 


\subsection{Palavras-chave de busca}

A escolha das palavras-chaves foi elaborada com base no método P.I.C.O. [8]. A sigla vem do inglês significando Population, Intervention, Comparison and Outcomes, no português, Polulação, Intervenção, Comparação e Resultados. Para a pesquisa realizada, o BIM foi definido como a população, uma vez que ele representa o principal assunto de interesse do estudo. Para a intervenção, que representa a situação de contorno, tem-se o VPL. Como o estudo buscou analisar o contexto geral no uso do VPL vinculado ao BIM, a comparação não se aplica e os resultados não foram restringidos, uma vez que objetiva-se com a pesquisa obter os efeitos dessa vinculção. Deste modo, as palavras-chaves definidas para este estudo foram: BIM e Building information; VPL e programação visual, no inglês, visual programming ou visual script.

\subsection{Base de dados}

A base de dados é a plataforma onde é feita a busca pelos artigos e textos científicos. Para esta pesquisa buscou-se a utilização das plataformas mais usuais dentro do contexto da engenharia civil: Scopus, Web of Science, Compendex e Science Direct.

\subsection{String de busca}

A string de busca é a configuração de como as palavras-chave serão associadas para formulação das pesquisas dentro das bases. Esta associação é feita através do operador boleano OR e do operador boleano AND. Deste modo, os strings de busca utilizados nesta pesquisa foram: (BIM OR "building information") AND (VPL OR "visual program*" OR "visual script*"). O sinal de asterisco $(*)$ foi utilizado com o intuito de obter todas as possíveis variações e derivações dos termos buscados, como visual program, visual programming, visual script e visual scripting.

\subsection{Critérios de seleção}

O primeiro filtro para seleção dos materiais utilizou-se das ferramentas de seleção disponibilizadas no próprio sistema das plataformas de pesquisa, onde se definiu o tipo de documento, restringindo-os a artigos publicados em periódicos e o período de retorno de 5 anos, ou seja, de novembro de 2014 a novembro de 2019.

O segundo filtro utilizado foi o de exclusão de textos duplicados. Como a pesquisa foi realizada em várias bases, existia a possibilidade de um mesmo documento estar vinculado a mais de uma. $\mathrm{O}$ terceiro filtro consistiu na eliminação dos artigos que não estivessem no idioma inglês ou português. Na quarta filtragem foi feita a leitura dos títulos, resumo e palavras-chave dos artigos, de modo a verificar os artigos aderentes ao tema pesquisado e no último filtro foi feita a verificação de gratuidade dos textos.

\subsection{Extração das informações}

Selecionados os estudos relevantes obtidos através do mapeamento, a leitura integral dos trabalhos foi feita de modo a identificar as principais contribuições de cada estudo e as lacunas de conhecimento existentes. Além disso, é feita também a categorização dos estudos.

Nesta etapa também foi realizada a amostragem por bola de neve (snowball sampling), que consistiu na leitura das referências bibliográficas dos trabalhos selecionados com o intuito de verificar a exixtência de outros estudos de interesse que não foram indentificados nas etapas anteriores e, portanto, não estavam presentes no mapeamento. 


\section{Resultados}

Os resultados referem-se à identificação dos principais estudos referentes à integração do VPL com o BIM, de modo a tentar identificar as principais áreas da construção civil que utilizam de forma conjunta estas duas ferramentas de trabalho.

A pesquisa realizada nas bases de dados retornou um total de 57 artigos, sendo a plataforma WEB OF SCIENCE e SCOPUS as que possuiram maior representatividade, apresentando um total $39 \%$ e $37 \%$, respectivamente, do total de resultados obtidos. A base com menos artigos encontrados foi a COMPENDEX, que retornou apenas 1 artigo, representado, por tanto, apenas $2 \%$ dos resultados.

Destes 57 artigos, 27 estavam duplicados, reduzindo os resultados de artigos aderentes para 30, sendo 1 destes no idioma italiano e, por tanto, desconsiderado da análise. A leitura dos títulos, resumos e palavras-chaves levou a exclusão de mais 1 artigo considerado não aderente a temática, levando, assim, a um resultado final de 28 artigos classificados. Dentre estes foi realizada a amostragem por bola de neve, porém não foram identificados novos artigos de interesse que não estivessem presentes no mapeamento já realizado.

Uma vez selecionados os artigos, foi feita a análise integral dos textos e a extração e categorização das informações obtidas. Em relação ao ano de publicação dos artigos, buscouse verificar o desenvolvimento e evolução do tema no meio acadêmico. Na Figura 1 é possível notar uma tendência crescente de publicações ao longo dos anos, principalmente a partir de 2017, indicando, também, como a preocupação da comunidade científica pelo assunto é relativamente recente. Esse interesse maior pela temática apenas nos últimos anos pode ser justificado pelo recente crescimetno e maior desenvolvilmento e uso da tecnologia da informação, não somente na área da construção civil, mas em diversos outros campos, de modo a automatizar, agilizar e facilitar os processos de trabalho.

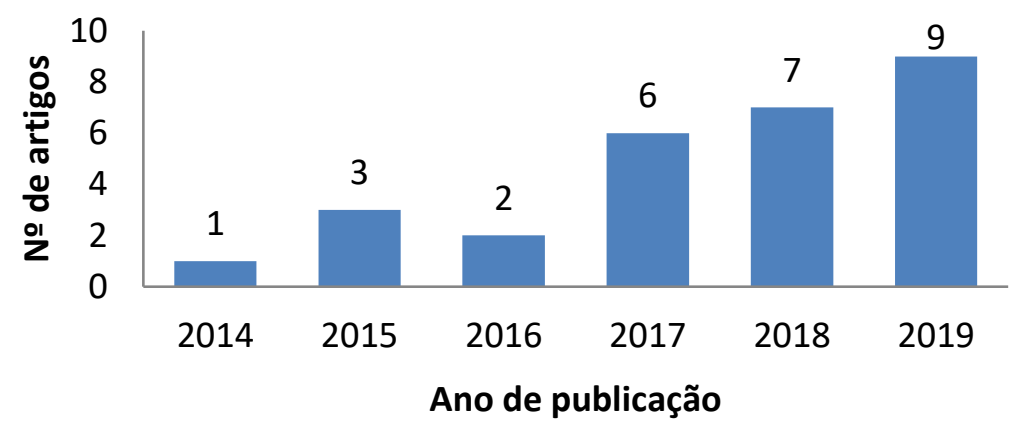

Figura 1: Distribuição dos artigos aderentes por ano de publicação (Fonte: Os autores).

Na Figura 2 é apresentada a distribuição continental de origem dos documentos que tratam sobre VPL aplicado no ambiente BIM. A maior percentagem de desenvolvimento se encontra na Europa (43\%), acompanhada, logo em seguida, pela Ásia (32\%). Estes resultados refletem o cenário existente quando se trata de temáticas inovadoras, onde existe uma tendência do tema começar a ser abordado com maior força no continente europeu e asiático, sendo difundido, subsequentemente, para os demais.

Em relação aos autores, dentre os 28 artigos aderentes, 4 são de autoria da pesquisadora Kensek, K., sendo esta a autora com maior número de publicações sobre a temática, com estudos que vem sendo realizados a pelo menos 5 anos (primeira publicação realizada em 2014). O segundo 
pesquisador com mais artigos publicados na área é o Borrmann, A., com 3 publicações aderentes e estudos sendo realizados desde 2015. Isto torna estes autores importantes e possíveis referências de destaque na temática.

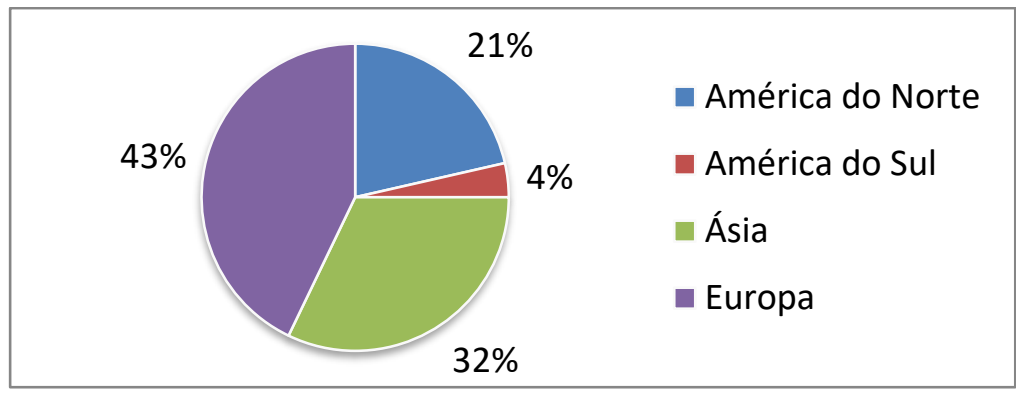

Figura 2: Distribuição continental dos artigos aderentes (Fonte: Os autores).

Com relação aos temas abordadas nos estudos, inicialmente foi feita a divisão dos artigos em 2 categorias:

- Categoria 1 - Artigos com o intuito em estudar e analisar diversas tématicas voltadas para o cenário da indústria de AEC e que utilizam o VPL integrado ao BIM como uma de suas ferramentas de trabalho ( $82 \%$ dos estudos);

- Categoria 2 - Artigos com o intuito em estudar e analisar, de modo geral, o uso do VPL dentro do contexto BIM (18\% dos estudos).

De modo a tentar identificar tendências no setor da construção civil de iniciativas envolvendo BIM-VPL, a categoria 1 foi analisada de forma mais profunda. Primeiramente foi realizada a identificação de quais etapas do processo construtivo (projeto, planejamento, execução, operação e manutenção, renovação e demolição) a integração do VPL com o BIM mais estava sendo utilizada (Tabela 1).

Tabela 1: Classificação dos artigos da categoria 1 quanto a etapa do processo construtivo

\begin{tabular}{lcc}
\hline Etapas & Quantidade de artigos & Percentual \\
\hline Projeto & 17 & $74 \%$ \\
Planejamento & 1 & $4 \%$ \\
Execução & 1 & $4 \%$ \\
Operação e manutenção & 1 & $4 \%$ \\
Renovação & 3 & $13 \%$ \\
\hline
\end{tabular}

Observa-se que não há o desenvolvimento da temática em todas as etapas, ficando a fase de demolição desfalcada de artigos aderentes. Ressalta-se também que a fase de projeto foi a que apresentou o maior foco de análises nos estudos (74\%). Dentro da construção civil, o projeto é um item de fundamental importância. É a partir dele que todo o resto se desenvolve. Além disso, um bom projeto é capaz de reduzir custos, minimizar possíveis imprevistos no decorrer 
da obra, garantir melhores condições de uso e manutenção, além de permitir construções mais sustentáveis e com maior nível de desempenho e eficiência energética. Desta forma, é natural que as pesquisas foquem nesta etapa construtiva, no entanto fica clara a existência de uma lacuna de conhecimento quanto à aplicação do conjunto BIM-VPL em outras etapas construtivas. Vale destacar que em relação à etapa de renovação, os artigos focaram na reconstrução virtual de patrimônios históricos e na gestão da informação, o chamado HBIM, que seria um modelo gráfico digital de informações sobre patrimônio.

A segunda análise feita com relação aos artigos da categoria 1 foi a classificação quanto as principais áreas de trabalho da construção civil (edificações, infraestrutura e transporte, geotecnia, hidráulica e saneamento) que as pesquisas estavam sendo desenvolvidas (Tabela 2). Dos 23 artigos, apenas 1 não aborda a temática de edificações, mostrando a existência de mais uma lacuna de conhecimento quanto a aplicação do conjunto BIM-VPL. O que poderia justificar este resultado seria o fato do BIM ser uma tecnologia pouco explorada dentro dessas outras áreas, o que refletiria, consequentemente, em poucos estudos integrando BIM e VPL. Vale ressaltar, no entanto, que para comprovar tal afirmativa, um levantamento a cerca da utilização do BIM dentro das diversas áreas da construção civil se faz necessário.

Tabela 2: Classificação dos artigos da categoria 1 quanto as áreas de trabalho da construção civil

\begin{tabular}{lcc}
\hline Áreas & Quantidade de artigos & Percentual \\
\hline Edificações & 22 & $96 \%$ \\
Geotecnia & 1 & $4 \%$ \\
\hline
\end{tabular}

Por último, e não menos importante, foi feita a classificação dos principais subtemas abordados nos artigos da categoria 1 (Tabela 3), onde foram identificados 13 subtemas distintos. É possível observar que o foco principal de aplicação da tecnologia BIM-VPL foi a análise de alternativas de design da edificação. Das 23 publicações, 8 trataram desta temática, onde a integração do BIM com o VPL permitiu que diferentes alternativas de design pudessem ser avaliadas de modo iterativo e integrado ao longo do processo de projeto. A programação visual aplicada dentro do contexto BIM permite ao profissional ver diretamente o efeito das alterações no modelo, o que tende a otimizar e acelerar o processo de tomadas de decisão, tornando os projetos mais assertivos e consistentes com os objetivos a qual se propõem.

Outro ponto forte no uso do BIM-VPL diz respeito a verificação de conformidade das construções com base em regras e requisitos pré-definidos (7 artigos) e a análise de eficiência energética e desempenho, principalmente desempenho térmico (5 artigos). No primeiro, o VPL é utilizado juntamente com o BIM para verificar se os projetos e construções atendem aos requisitos normativos, requisitos de projeto, do cliente, entre outros [9], [10], [11], [12], [13], [14], [15].

No que diz respeito à análise de eficiência energética e desempenho, um dos estudos buscou a criação de uma estrutura de otimização do desempenho visando à minimização do consumo de energia [16]. Outro artigo buscou avaliar o desempenho energético (uso de energia do edifício) com base na variabilidade de ocupação dos usuários [17]. Também foram realizados estudos buscando a avaliação de desempenho do envelope das edificações de acordo com normativas específicas [18], [12]. E por fim, um dos artigos trata sobre a análise de desempenho utilizando 
a termografia como ferramenta para identificar as causas-raiz dos problemas térmicos e as ineficiências dos envelopes de construção [19]. De modo geral estes estudos demostraram a importância e o potencial da integração do BIM e do VPL para o acesso as informações armazenadas no modelo BIM, no auxílio aos processos de simulação e para a avaliação de eficiência energética e desempenho.

Tabela 3: Classificação dos artigos da categoria 1 quanto aos principais subtemas abordados

\begin{tabular}{lc}
\hline Subtemas & Quantidade de artigos \\
\hline Alternativas de design da edificação & 8 \\
Verificação de conformidade das construções com base em & 7 \\
regras/requisitos & 5 \\
Eficiência energética/Desempenho & 4 \\
Avaliação/Custos do ciclo de vida & 3 \\
Sistema de Informações do patrimônio construído & 2 \\
Integração de sensores ambientais ao BIM & 1 \\
Gerenciamento de canteiro de obras & 1 \\
Avaliação do Índice de Uso de Concreto (CUI) & 1 \\
Avaliação do nível de flexibilidade dos edifícios & 1 \\
Planejamento da segurança para trabalhos de escavação & 1 \\
Avaliação dos arredores da construção & 1 \\
Conforto térmico & 1 \\
Sistemas de fachadas inteligentes & 1 \\
\hline
\end{tabular}

Além dos subtemas já citados, vale destacar também que dentro do período de abrangência do mapeamento, dos 13 subtemas identificados, mais da metade foi estudada uma única vez. Com isso, é possível perceber que a vinculação do BIM com a programação visual possui uma gama grande de aplicações dentro da construção civil, mas que ainda foram muito pouco exploradas. Existem lacunas de conhecimento no contexto do BIM-VPL que abrem as portas para potenciais estudos em diversos campos, tais como a análise de conforto térmico por meio dos métodos de voto médio predito (PMV) e percentagem de pessoas insatisfeitas (PPD); abranger diferentes tipos de regras e requisitos para as várias etapas do ciclo de vida do edifício e não apenas na fase de projeto, verificar o comportamento do BIM-VPL quando submetidos a análise de alternativas de design da edificação baseado não apenas em um único citério, mas em vários critérios de decisão, como análise conjunta de desempenho térmico, avaliação do ciclo de vida e custos de construção; integrar o BIM-VPL com outras tecnologias, como a de realidade aumentada; entre outros.

Por fim, partindo para uma análise da tecnologia BIM-VPL em si, o que inclui a análise dos artigos da categoria 2, tanto as empresas de software quanto os programadores entusiastas têm 
desenvolvido um conjunto de componentes que aprimoram essas ferramentas gráficas inovadoras. Usuários de iniciantes a avançados podem encontrar utilidade nessas ferramentas. O primeiro passo é aprender o básico do software e entender sua aplicabilidade aos problemas e oportunidades que surgem no design, construção e operação da construção [20]. Partindo disso, foi verificado que o VPL tem um alto grau de facilidade de uso e geralmente reduz o obstáculo para que os usuários que não são da área de tecnologia entrem no campo da programação [21]. O uso do VPL para manipular os dados BIM pode levar o usuário a automatizar tarefas de trabalho, desenvolvendo programas personalizados. Além disso, o mesmo programa pode ser desenvolvido de várias maneiras, dependendo do nó/fluxo de trabalho que o usuário implementa [12]. A programação visual, se bem feita, pode garantir que um parâmetro específico possa ser facilmente alterado para observar seu efeito imediato nos resultados gerais da simulação, fornecendo um link entre a geometria e os dados, além de permitir que um fluxo de trabalho, uma vez desenvolvido, possa ser facilmente usado para estudar outros edifícios [7].

Com relação às desvantagens, as pesquisas indicam que a escalabilidade (capacidade de lidar com grandes quantidades de informações) dos programas criados com VPL pode ser um problema para essa abordagem [22]. Em particular, fluxos de controle mais complexos, como loops, geralmente não podem ser implementados ou são muito grandes e difíceis de entender [21]. Outro ponto de destaque é que o fluxo de trabalho com BIM-VPL não pode evitar a fase de preparação do modelo BIM onde o modelo precisa ter sido enriquecido com as informações semânticas corretas de modo que os dados necessários para o funcionamento correto dos scripts estejam bem estruturados e definidos [23].

\section{Conclusões}

Esse artigo apresentou como objetivo identificar as tendências no setor da construção civil no que se refere a utilização do VPL dentro do contexto BIM, além de identificar o estado da arte no que se refere a esta abordagem. Para cumprir tal objetivo foi realizado um MSL, sendo levantada uma amostra de artigos nos últimos 5 anos.

A análise dos dados possibilitou concluir que atualmente os estudos na área se dividem em artigos focados apenas em estudar a integração das duas tecnologias em si e nas pesquisas que utilizaram essa vinculação como uma ferramenta para realizar estudos diversos dentro do contexto da indústria de AEC. Para o segundo caso, foi verificado como se dava a utilização do BIM-VPL em relação as principais etapas do processo construtivo, dentro das principais áreas de trabalho da construção civil e ainda a classificação dos principais subtemas estudados utilizando de forma conjunta estas duas tecnologias.

A partir disto foi observado, primeiramente, que o uso conjunto do BIM com o VPL é um tema contemporâneo que possui como foco de atuação a área de edificações. Segundo, as pesquisas são voltadas principalmente para a etapa de projetos. Terceiro, dentre os 13 subtemas de trabalho identificados, os principais estudos de interesse são voltados para a análise de alternativas de design da edificação e verificação de conformidade das construções com base em regras e requisitos.

De modo geral, o mapeamento permitiu verificar que existem áreas e temáticas especifícas nos quais o conjunto BIM-VPL vem sendo mais aplicado, mas que também existe uma gama 
potencial de aplicações dentro da construção civil que ainda foram pouco exploradas, proporcionando um leque bastante grande e diversificado de pesquisas a serem desenvolvidas. Como principais contribuições deste estudo destaca-se a percepção de que a incorporação do VPL ao BIM não é apenas possível, mas se tornará cada vez mais comum na academia e no mercado de trabalho. Esta integração permite que estudos e análises personalizadaos sejam realizados pelas empressas e profissionais da área de AEC, permitindo o acesso direto e de modo mais eficiente aos dados e parâmetros da construção a partir da elaboração de algoritmos personalizados e especificos ao objetivo do trabalho que se deseja desenvolver.

Além disso, este estudo comprova que os profissionais da construção são capazes de otimizar os seus trabalhos por meio do desenvolvimento de suas próprias ferramentas usando BIM e VPL, em vez de depender completamente de ferramentas comerciais.

Por fim, cabe destacar que apesar das vantagens apresentadas, a depender da complexidade dos probelmas a serem solucionados, a programação visual pode não ser a opção mais viável, fazendo com que o aprendizado da programação textual, apesar de levar mais tempo e esforço do que aprender um VPL, seja a escolha mais adequada e um possível caminho a ser adotado.

\section{Referências}

[1] ISO 29481-1:2010, "ISO 29481-1:2010 Building information modelling — Information delivery manual - Part 1: Methodology and format," International Organization for Standardization (ISO), 2010.

[2] D. V. S. Baia, "Uso de ferramentas BIM para o planejamento de obras da construção civil," Dissertação de Mestrado, Universidade de Brasília, Brasília, Distrito Federal, Brasil, 2015.

[3] T. F. Campestrini, M. C. Garrido, R. M. Jr., S. Scheer, and M. do C. D. Freitas, Entendendo BIM. Curitiba, PR: Universidade Federal do Paraná, 2015.

[4] M. Marzouk and A. Abdelaty, "Monitoring thermal comfort in subways using building information modeling," Energy and Buildings, vol. 84, pp. 252-257, Aug. 2014. doi: 10.1016/j.enbuild.2014.08.006

[5] A. Leitão and L. Santos, "Programming Languages for Generative design - Visual or Textual?," in Respecting Fragile Places: 29th eCAADe Conference, Liubliana, Liubliana, Eslovênia, 2011, pp. 549-557.

[6] P. H. Gonçalves, "Automatização do fluxo de informações dentro do processo Bim com foco na avaliação do desempenho térmico, acústico e o custo das decisões projetuais," Tese de Doutorado, Universidade de Brasília, Brasília, Ditrito Federal, Brasil, 2018.

[7] K. Kensek, "Visual programming for building information modeling: Energy and shading analysis case studies," Journal of Green Building, vol. 10, pp. 28-43, Nov. 2015. doi: 10.3992/jgb.10.4.28

[8] H. L. Agostinho and A. D. Granja, "Comparação de Modelos Contratuais na Construção Civil: Um Mapeamento Sistemático de Literatura," in XVI Encontro Nacional de Tecnologia do Ambiente Construído, São Paulo, São Paulo, Brasil, 2016, pp. 3476-3488.

[9] M. M. Singh, A. Sawhney, and A. Borrmann, "Modular Coordination and BIM: Development of Rule Based Smart Building Components," Procedia Engineering, vol. 123, pp. 519-527, Out. 2015. doi: 10.1016/j.proeng.2015.10.104

[10] J. Dimyadi, W. Solihin, C. Preidel, and A. Borrmann, "Towards code compliance checking on the basis of a visual programming language," Journal of Information Technology in 
Construction, vol. 21, pp. 402-421, 2016.

[11] D. M. dos Santos and J. N. Beirão, "Parametrical design tool and the production of technical data for superadobe domes," Gestão \& Tecnologia de Projetos, vol. 14, pp. 45-60, Set. 2019. doi: 10.11606/gtp.v14i1.148182

[12] T. E. Seghier, Y. W. Lim, M. H. Ahmad, and W. O. Samuel, "Building Envelope Thermal Performance Assessment Using Visual Programming and BIM, based on ETTV requirement of Green Mark and GreenRE," International Journal of Built Environment and Sustainability, vol. 4, pp. 227-235, Set. 2017. doi: 10.11113/ijbes.v4.n3.216

[13] S.-L. Fan, H.-L. Chi, and P.-Q. Pan, "Rule checking Interface development between building information model and end user," Automation in Construction, vol. 105, p. 102842, Set. 2019. doi: 10.1016/j.autcon.2019.102842

[14] P. Ghannad, Y.-C. Lee, J. Dimyadi, and W. Solihin, "Automated BIM data validation integrating open-standard schema with visual programming language," Advanced Engineering Informatics, vol. 40, pp. 14-28, Abr. 2019. doi: 10.1016/j.aei.2019.01.006

[15] J. Li, N. Li, K. Afsari, J. Peng, Z. Wu, and H. Cui, "Integration of Building Information Modeling and Web Service Application Programming Interface for assessing building surroundings in early design stages," Building and Environment, vol. 153, pp. 91-100, Abr. 2019. doi: 10.1016/j.buildenv.2019.02.024

[16] M. Rahmani Asl, S. Zarrinmehr, M. Bergin, and W. Yan, "BPOpt: A framework for BIMbased performance optimization," Energy and Buildings, vol. 108, pp. 401-412, Dez. 2015. doi: 10.1016/j.enbuild.2015.09.011

[17] A. Zani, L. C. Tagliabue, T. Poli, A. L. C. Ciribini, E. De Angelis, and M. Manfren, "Occupancy Profile Variation Analyzed through Generative Modelling to Control Building Energy Behavior," Procedia Engineering, vol. 180, pp. 1495-1505, Mai. 2017. doi: 10.1016/j.proeng.2017.04.312

[18] W. Natephra, N. Yabuki, and T. Fukuda, "Optimizing the evaluation of building envelope design for thermal performance using a BIM-based overall thermal transfer value calculation," Building and Environment, vol. 136, pp. 128-145, Mai. 2018. doi: 10.1016/j.buildenv.2018.03.032

[19] W. Natephra, A. Motamedi, N. Yabuki, and T. Fukuda, "Integrating 4D thermal information with BIM for building envelope thermal performance analysis and thermal comfort evaluation in naturally ventilated environments," Building and Environment, vol. 124, pp. 194-208, Nov. 2017. doi: 10.1016/j.buildenv.2017.08.004

[20] K. M. Kensek, "Teaching visual scripting in BIM: A case study using a panel controlled by solar angles," Journal of Green Building, vol. 13, pp. 113-138, Jan. 2018. doi: 10.3992/1943-4618.13.1.113

[21] C. Preidel, S. Daum, and A. Borrmann, "Data retrieval from building information models based on visual programming," Visualization in Engineering, vol. 5, p. 18, Dez. 2017. doi: 10.1186/s40327-017-0055-0

[22] T. Wortmann and B. Tunçer, "Differentiating parametric design: Digital workflows in contemporary architecture and construction," Design Studies, vol. 52, pp. 173-197, Set. 2017. doi: 10.1016/j.destud.2017.05.004

[23] C. Cavalliere, G. R. Dell'Osso, F. Favia, and M. Lovicario, "BIM-based assessment metrics for the functional flexibility of building designs," Automation in Construction, vol. 107, p. 102925, Nov. 2019. doi: 10.1016/j.autcon.2019.102925 